\title{
¿Qué hay de nuevo en calcifilaxia?
}

\author{
¿What is new in calciphylaxis?
}

\section{¿O que há de novo na calcifilaxia?}

\author{
Juan Carlos Restrepo Medrano* \\ Sherezada Suaza Velásquez ${ }^{* *}$
}

\section{Resumen}

Objetivo: Revisar la literatura científica publicada en las principales bases de datos, entre los años 1962 y 2016, cuyo objetivo fue abordar el tema de la calcifilaxia y sus principales características. Metodología: Se llevó a cabo una revisión de la literatura en las principales bases de datos de las Ciencias de la Salud y las Ciencias Sociales (medline-pubmed, cinhal, web of science, lilacs, sociological abstracts, cuiden, embase, psycoinfo e isi web of knowledge). Se emplearon descriptores en español, inglés y portugués: Calcifilaxis, diálisis, hiperparatiroidismo, necrosis, gangrena, calcificación vascular, cuidados de enfermería y diagnósticos de enfermería. Se hizo una revisión sistemática de los artículos seleccionados utilizando un protocolo para extraer los datos. Resultado: Se encontraron 45 artículos que cumplían con los criterios de búsqueda establecidos, 35 reportaron los resultados de ensayos clínicos o estudios cualitativos, 4 correspondían a revisiones sistemáticas y 6 se catalogaron como estudios retrospectivos. Los autores señalaron las principales características de la enfermedad, de los pacientes y los tratamientos más usados, pero poco del cuidado que debe brindar el profesional enfermero. Conclusión: En los estudios analizados se identificaron principalmente aspectos sobre el curso de la enfermedad y el tratamiento; se reportó la efectividad de algunos tratamientos utilizadas en la atención de dichos pacientes, pero ninguna de ellas definitiva, lo que permite proponer algunas acciones desde la disciplina enfermera.

Palabras clave: Arteriolopatía urémica calcificante, necrosis cutánea, calcificación microvascular

\section{Abstract}

Objective: To analyze the scientific literature published in the main databases between 1962 and 2016, whose objective was to address the issue of calciphylaxis and its main characteristics? Methodology: A literature review was carried out in the main databases of the Health Sciences and Social Sciences (medline-pubmed, cinhal, web of science, lilacs, sociological abstracts, cuiden, embase, psycoinfo and isi web of knowledge). An analytical review of the selected articles was made using a protocol to extract the data. Results: 45 articles were found that fulfilled the established search criteria, 35 reported the results of clinical trials or qualitative studies, 4 corresponded to systematic reviews and 6 were classified as retrospective studies. The authors pointed out the main characteristics of the disease, the patients and the most used treatments, but little of the care that should be provided by the nurse practitioner. Conclusion: In the analyzed studies, aspects regarding the course of the disease and treatment were identified; it was reported the effectiveness of some treatments used in the care of these patients, but none of them definitive, which allows to propose some actions from the nurse discipline.

Key words: Calciphylaxis, nurse, Dialysis, chronic ulcers

\section{Para citar este artículo/ To reference this article / Para citar este artigo/}

Restrepo Medrano JC, Suaza Velásquez S. ¿Qué hay de nuevo en calcifilaxia?. Rev. cienc. cuidad. 2018;15(1):110-122 Este es un artículo bajo la licencia CC BY (https://creativecommons.org/licenses/by/4.0/) @ ) 
Objetivo: Revisar a literatura científica publicada nas principais bases de dados, entre os anos $1962 \mathrm{e}$ 2016, cujo objetivo era abordar o tema da calcifilaxia e suas principais características. Metodologia: Uma revisão da literatura foi realizada nas principais bases de dados das Ciências da Saúde e das Ciências Sociais (Medline-Pubmed, Cinhal, Web of Science, Lilacs, Sociological abstracts, Cuiden, Embase, Psycoinfo e Isi Web of Knowledge). Foram usados descritores em espanhol, inglês e português: calcifilaxia, diálise, hiperparatiroidismo, necrose, gangrena, calcificação vascular, cuidados de enfermagem e diagnósticos de enfermagem. Foi feita uma revisão sistemática dos artigos selecionados utilizando um protocolo para extrair os dados. Resultados: Encontraram-se 45 artigos que cumpriam com os critérios de busca estabelecidos, 35 reportaram os resultados de ensaios clínicos ou estudos qualitativos, quatro (4) correspondiam a revisões sistemáticas e seis (6) se catalogaram como estudos retrospectivos. Os autores apontaram as principais características da doença, dos pacientes e os tratamentos mais usados, mas pouco do cuidado que debe oferecer o professional da enfermagem. Conclusão: Nos estudos analisados se identificaram principalmente aspectos sobre o curso da doença e o tratamento; se reportou a efetividade de alguns tratamentos utilizados no atendimento desses pacientes, mas nenhum deles definitivo, o que permite propor algumas ações desde a profissão da enfermagem.

Palavras-chave: Arteriopatia urêmica calcificante, necrose cutânea, calcificação microvascular

\section{Introducción}

La calcifilaxia es una alteración vascular rara, aunque grave, con una elevada tasa de morbimortalidad y que se caracteriza por una progresiva calcificación vascular con necrosis isquémica de la piel y de los tejidos blandos. Los primeros casos fueron descritos en la década de los 60; cuenta con una prevalencia que oscila entre el 1 y el $4 \%$ de los pacientes con insuficiencia renal crónica, en programa periódico de sustitución mediante hemodiálisis o trasplante renal, siendo excepcional en los pacientes sometidos a diálisis peritoneal o en estadios pre diálisis (1). Con frecuencia, afecta a pacientes mayores, de sexo femenino, de raza blanca, diabéticos y VIH positivos.

El término calcifilaxis fue acuñado por Seyle en 1962 (1) para describir un fenómeno puramente experimental observado en ratas, y lo definió como un estado de hipersensibilidad sistémica en el cual los tejidos responden frente a agentes desencadenantes concretos con un rápido depósito de calcio. Estos animales fueron «sensibilizados» por vitamina $\mathrm{D}$, hormona paratiroidea (PTH) y diversas «injurias» nefrotóxicas; posteriormente fueron tratados con diversos productos («provocadores») que indujeron una inflamación y calcificación en casi todos los órganos examinados, incluyendo la piel.

La patogénesis de esta enfermedad aún no es clara; sin embargo, se han involucrado diversos factores de riesgo, destacando el hiperparatiroidismo secundario con una elevación del producto calcio $(\mathrm{Ca})$ - fosforo (P), superior a $55 \mathrm{mg} / \mathrm{dl}$, niveles normales o elevados de Ca en sangre y altas dosis de metabolitos activos de la vitamina $\mathrm{D}$ (2-4). Otros posibles factores de riesgo son la deficiencia de proteína $\mathrm{C}$ y $\mathrm{S}$, anticoagulantes, antagonistas de la vitamina $\mathrm{K}$, acumulación de $\mathrm{Ca}$ sobre las valvas del corazón, hipertensión arterial y dislipidemia, es decir, toda una serie de factores cuya consecuencia final es una afectación vascular.

Las alteraciones del metabolismo Ca - P y los niveles elevados de la hormona paratiroides (PTH) están íntimamente relacionadas con el desarrollo de la calcifilaxis. A pesar de esto, diversos estudios (4) demuestran que no necesariamente deben estar alterados dichos parámetros ni se correlacionan con la severidad de la enfermedad.

Los pacientes sin enfermedad renal con calcifilaxis, que presentan niveles de PTH en sangre superiores a $200 \mathrm{pg} / \mathrm{ml}$, sobre todo si no responden al tratamiento médico, suelen beneficiarse de la paratiroidectomía; en cambio, los pacientes con enfermedad renal crónica con hiperparatiroidismo severo (PTH $>750 \mathrm{pg} /$ $\mathrm{ml}$ ) y que no responden al tratamiento médico (calcimiméticos, análogos de la vitamina $\mathrm{D}$, quelantes, etc.), son subsidiarios de tratamiento quirúrgico mediante paratiroidectomía (5). Sacharías et al. (6) concluyen que el uso de sales cálcicas, probablemente, 
ISSN-PRINT

1794-9831

E-ISSN 2322-7028

Vol. 15 No. 1

Ene - Jun 2018

Cúcuta, Colombia contribuye a un aumento en la incidencia de la calcifilaxis. Por ello, hay en el mercado quelantes del fósforo $(\mathrm{P})$ que no aportan calcio $(\mathrm{Ca})$ como el carbonato de lantano y el sevelamer, entre otros, que estarían indicados para este tipo de pacientes, evitando el uso de quelantes cálcicos.

Otro factor de riesgo implicado es la obesidad (7). Como sustento de esto se encuentra el estudio de Bleyer et al. (8), en el que se valoran nueve pacientes con calcifilaxis proximal y se identifica como factor de riesgo un elevado índice de masa corporal $(35 \mathrm{~kg} /$ $\mathrm{m}^{2}$ ). La razón por la cual se relaciona la obesidad mórbida con la calcifilaxis es, probablemente, la mayor cantidad de tejido adiposo que está en contacto con la circulación sanguínea, más frecuente en la mujer (9) -en particular a nivel de las caderas, las nalgas, el área infraumbilical y la parte superior de los muslos-, ya que es en esas partes del cuerpo donde se localiza la mayor cantidad de depósitos grasos. Así mismo, la calcifilaxis se ha presentado en zonas de punción con insulina subcutánea o heparina (10).

De igual manera, los niveles bajos de albúmina sérica se relacionan con la calcifilaxis, principalmente cuando su localización es proximal y no implica, necesariamente, la malnutrición como factor de riesgo (6). La raza blanca, los traumatismos, la administración de warfarina, hierro intravenoso, prednisona (en modelos experimentales) enfermedad (11-12), así como la terapia con rayos ultravioleta en un paciente con enfermedad renal crónica estadio $\mathrm{V}$ y secundaria a lupus eritematoso sistémico con prurito intratable, también se han evidenciado como factor de riesgo (13).

De otro lado, las alteraciones metabólicas y tratamientos asociados con la insuficiencia renal crónica no explican, por sí solos, el desarrollo de esta patología. Se especula que mecanismos moleculares más complejos subyacen al desarrollo de este trastorno, entre ellos las deficiencias de los inhibidores de la calcificación vascular (fetuin-A y la proteína Gla de la matriz) y la alteración de vías moleculares implicadas en la regulación de la mineralización extraesquelética, como la vía del factor nuclear $\kappa \mathrm{B}$ (receptor activador RANK, ligando RANK y la osteoprotegerina) $(1,5)$, que está íntimamente relacionada con la mineralización ósea y periférica. La proteína Gla de la matriz ósea inhibe la acción de múltiples proteínas, alterando la capacidad de unión y depósito de calcio en la matriz ósea extracelular (4). El fetuin A es un importante inhibidor circulante de la calcificación y se disminuye durante las fases inflamatorias persistentes. Datos serológicos en pacientes con tiempos prolongados de diálisis y bajas concentraciones del fetuin A, han mostrado precipitación vascular de calcio y fósforo, lo que aparentemente afecta la resolución de la inflamación, modulando la fagocitosis de células apoptóticas por macrófagos (5).

Las lesiones cutáneas de calcifilaxis se presentan como una serie de nódulos subcutáneos y placas violáceas, purpúricas o equimóticas, extremadamente dolorosas, sobre una base de eritema moteado o reticular, semejante a la livedo reticularis. Estas lesiones progresan en pocos días a úlceras necróticas recubiertas de escaras negruzcas (14).

Existen dos formas diferentes de presentación clínica: la calcifilaxis acra y la proximal; ambas se distribuyen preferentemente por las extremidades. En la distal o acra (15), como forma más frecuente, se afectan las piernas, los pies y los dedos; la forma proximal es menos frecuente pero de peor pronóstico y afecta los muslos, las nalgas y el abdomen. Los diferentes estudios relacionan la calcifilaxis proximal con los pacientes diabéticos, siendo las alteraciones del metabolismo Ca-P menos severas que en los pacientes con calcifilaxis acra y, no obstante, tienen un peor pronóstico (15).

Para realizar el diagnóstico de la enfermedad, el primer paso lo constituyen la anamnesis y el examen físico (dolor, presencia de lesiones ulceradas dolorosas, etc.) y se confirma con el estudio radiológico e histológico (16). El procedimiento más sensible para detectar la calcifilaxis es la realización de una radiografía usando la técnica de la mamografía. La gammagrafía ósea se ha mostrado como una técnica diagnóstica muy sensible, observándose una hipercaptación subcutánea del isótopo (tecnecio 99), característica de la calcifilaxis, correspondiente a las placas calcificadas. De ser posible, se debe evitar la biopsia de la lesión cutánea, ya que puede precipitar su ulceración y posterior infección, con el consecuente riesgo de septicemia. Además, los hallazgos histopatológicos pueden ser inespecíficos (17). 
El presente estudio tiene como objetivo hacer una revisión analítica de la literatura científica existente sobre la calcifilaxia, sus principales características, tratamiento y las intervenciones de los profesionales de enfermería en el desarrollo de la misma.

\section{Metodología}

Se realizó un análisis de las artículos existentes sobre el tema de calcifilaxis, publicados en las bases de datos de ciencias sociales y de salud: Medline (Pubmed), Cinhal, Web of Science, Lilacs, Sociological Abstracts, Cuiden, Embase, PsycoInfo e ISI web of Knowledge. El periodo analizado comprendíó publicaciones desde el inicio de cada base de datos hasta junio de 2016 y se emplearon los siguientes descriptores en español, inglés y portugués: calcifilaxis, diálisis, hiperparatiroidismo, necrosis, gangrena, calcificación vascular, cuidados de enfermería y diagnósticos de enfermería. Para precisar la búsqueda, se usaron expresiones alternativas como prevención de heridas, herida crónica y cicatrización, existentes en los títulos, en los resúmenes o en textos completos.

Los artículos fueron seleccionados teniendo como criterio el hecho de que entre los objetivos se abordara el cuidado de heridas crónicas en el paciente con calcifilaxia. Se excluyeron artículos sin resúmenes disponibles, editoriales, ponencias en congresos, reporte de experimentos en animales o estudios escritos en idiomas diferentes a los establecidos. Los textos se analizaron mediante un protocolo ad hoc, realizado exclusivamente para este estudio, en el cual se recogían los datos más relevantes de cada uno de los artículos encontrados (revista, tipo de artículo, año, metodología, resultados, etc.). Este protocolo fue revisado en dos ocasiones por los investigadores, con el fin de evitar sesgos en el trabajo y asegurar la toma correcta de los datos. Para valorar la calidad de la evidencia y la fuerza de las recomendaciones en los artículos, se empleó el sistema GRADE (Grading of Recommendations Assessment, Development and Evaluation Working Group), que proporciona una descripción detallada de los criterios de calidad de evidencia de los artículos científicos encontrados, respecto a resultados o desenlaces concretos $\mathrm{y}$, además, usa definiciones explícitas y juicios secuenciales durante el proceso de categorización y separa la calidad de la evidencia de la fuerza de las recomendaciones (18).

\section{Resultados}

Se encontraron 45 artículos que cumplían con los criterios establecidos: 35 reportaron los resultados de ensayos clínicos o estudios cualitativos, cuatro correspondían a revisiones sistemáticas y seis se catalogaron como estudios retrospectivos. A continuación se describen sus aspectos relevantes:

\section{Tipos de estudio}

Los artículos que reportaron ensayos clínicos compartían características de la enfermedad, como el inicio de la misma, la epidemiología y algunas partes del tratamiento (6-7, 9-11, 19-20). La mayoría de ellos concuerdan con la alta mortalidad que presenta esta enfermedad, la cual está por encima del 50 $\%$, en ninguno se establece el papel de la hormona paratiroidea, pero sí se establece que los pacientes cuentan con un trastorno óseo mineral. Dentro de los casos analizados se relaciona, de forma directa, la desregulación del metabolismo óseo mineral, incluyendo la hiperfosfatemia, el hiperparatiroidismo y el producto fosfocálcico elevado, el cual, según distintos autores, es una condición importante para desencadenar la enfermedad (21).

\section{Origen de la enfermedad}

Los artículos analizados tienden a señalar un origen multifactorial de la enfermedad, muchos de ellos basados en la hipótesis del rol de la proteína $\mathrm{C}$, cuya deficiencia lleva a un estado de hipercoagulabilidad que induce a trombosis en los pequeños vasos y desencadena los eventos de isquemia-necrosisgangrena-sepsis (22). Otros mecanismos que explican la necrosis de la piel incluyen la deficiencia de la proteína S (23) y la injuria endotelial producida por agentes tales como los gérmenes gram negativos. La heparina puede contribuir a la cascada de eventos como la mala absorción y un inadecuado aporte de vitamina $\mathrm{K}$. Adicionalmente, estudios recientes encontraron que las células musculares lisas son responsables de la producción de calcificación intravascular, la cual estaría asociada con la expresión de una proteína productora de matriz ósea denominada "osteopontina" (24).

Las alteraciones en la producción de vitamina $\mathrm{D}$, que ocurren en los pacientes con insuficiencia renal crónica, reducen la absorción intestinal de calcio 
ISSN-PRINT

1794-9831

E-ISSN 2322-7028

Vol. 15 No. 1

Ene - Jun 2018

Cúcuta, Colombia que se expresa como hipocalcemia, lo que estimula la producción de paratohormona, provocando hiperparatiroidismo secundario que intensifica la absorción ósea con el fin de revertir la hipocalcemia. Sin embargo, esto origina hiperfosfatemia, patología que se agrava por la disminución del aclaramiento renal de fosfatos presentes en los pacientes con insuficiencia renal crónica. Estas alteraciones en el metabolismo del fósforo y el calcio pueden provocar calcificaciones metastásicas, en especial si la relación $\mathrm{Ca}++/ \mathrm{P}$ es mayor a 70; sin embargo, existen pacientes que tienen calcifilaxis con relación de $\mathrm{Ca}++/ \mathrm{P}$ menores y concentraciones normales de paratohormona (5).

\section{Deficiencias nutricionales}

Seguido de lo anterior, en varios estudios los pacientes son diagnosticados con problemas graves de desnutrición, pero no se establece esta característica como necesaria para desencadenar la enfermedad. De la misma manera, se describieron como condiciones necesarias para desencadenar esta patología la presencia de enfermedades renales; sin embargo, en algunos estudios se describen pacientes con trastornos no urémicos, presentándose sobre todo en mujeres con alguna deficiencia en la vitamina $\mathrm{D}$, pérdida de peso y enfermedad renal no crónica (25). Llama la atención, además, que muchos de los pacientes diagnosticados con esta enfermedad no recibieron tiosulfato de sodio, cinacalcet o terapia de oxígeno hiperbárico, a pesar de ser uno de los tratamientos a elección reportados en la bibliografía encontrada (26-27).

\section{Lesiones}

En cuanto a las lesiones que causa la enfermedad, diversos estudios realizan un seguimiento de este fenómeno, observando que, después del diagnóstico inicial, estas lesiones cerraban entre 3 meses y 1 año después, si el paciente reportaba valores normales de calcio y fósforo, entre otros. Así mismo, se reportó cómo a varios pacientes fue necesario realizarles exéresis quirúrgica, sin obtener mucha mejoría, pero aumentando la frecuencia de las diálisis $\mathrm{y}$, por ende, la concentración del calcio del baño de diálisis a 1,5 -1 mEq (28-29). En los estudios analizados es común el inicio de las lesiones, aunque se reportó también que en algunos pacientes pueden comenzar con prurito, pápulas o nódulos eritematosos que luego evolucionan de forma rápida a úlceras de tipo necrótico, secundarias a la calcificación de pequeños vasos y necrosis tisular. Es característico el intenso dolor que ocasionan estas lesiones, las cuales presentan una tendencia a desarrollarse en zonas con antecedentes de trauma previo, especialmente en miembros inferiores $(90 \%)$, principalmente tobillos y piernas. Cuando la afectación es proximal (muslos, nalgas, abdomen, pecho, genitales y hombros) se asocia con peor pronóstico (30-34).

\section{Diagnóstico diferencial}

Al realizar el diagnóstico diferencial de la enfermedad se observa que los más importantes fueron: úlceras vasculares, vasculitis, pioderma gangrenoso, oxapioderma gangrenoso, oxalosis, calcificación metastásica, calcificación idiopática, calcificación distrófica, pseudoxantoma elástico, criofibrinogenemia y coagulación intravascular diseminada (35-36).

De los anteriores, los más representativos y que más se asemejan a la calcifilaxia son:

- Oxalosis: es la cristalización sistémica de oxalato de calcio en huesos, miocardio, tiroides, SNC, vasos sanguíneos y menos frecuentemente en la piel. Se observa en pacientes sometidos a hemodiálisis y con insuficiencia renal crónica. Las manifestaciones clínicas incluyen livedo reticularis, con placas violáceas dolorosas que pueden evolucionar a necrosis tisular, escaras negruzcas que no cicatrizan, gangrenas o nódulos subungueales y pequeñas pápulas en el pulpejo de los dedos.

- Procesos vasculíticos

\section{- Pioderma gangrenoso}

- Pseudoxantoma elástico: condición hereditaria en la que existe una alteración en el tejido elástico que conduce a alteraciones en la retina, la piel y órganos viscerales. Las alteraciones de la piel pueden consistir en placas semejantes a las de la calcifilaxis, que se acompañan de calcificaciones en los tejidos blandos (37).

- Púrpura fulminans: la presentación clínica y el curso de la enfermedad son indistinguibles de la calcifilaxis $\mathrm{y}$ ocurre en deficiencia hereditaria o adquirida de proteínas anticoagulantes, proteína C y S (38). 
- Criofibrinogenemia: está asociada con enfermedades del colágeno, enfermedades malignas metastásicas y alteraciones tromboembólicas en las vísceras y en la piel, en donde puede producir necrosis cutánea. Su etiología es desconocida, pudiendo producirse por inhibición de la fibrinólisis, acumulándose de esta manera el criofibrinógeno. También se ha observado en pacientes con diálisis crónica e insuficiencia renal terminal.

En algunos de los estudios analizados se han reportado adenomas paratiroideos y antecedentes de niveles elevados de PTH en pacientes con calcifilaxis. La elevación persistente de los niveles de PTH por encima de $800 \mathrm{pg} / \mathrm{ml}$ se relaciona, según los hallazgos, con aumento del riesgo de calcificación vascular. Sin embargo, no se ha establecido la relación entre los niveles elevados de PTH y la fisiopatología de la patología dentro de ninguno de los estudios (26).

\section{Imagenologia}

Respecto a los estudios de imagen, en las investigaciones analizadas se ha sugerido desde la realización de ultrasonido hasta la arteriografía. Recientemente se ha propuesto el uso de la tomografía computarizada para el diagnóstico temprano de esta entidad, que se sugiere al realizar cortes pre contraste donde se observan calcificaciones en las arterias comprometidas, así como un área del glande que no capta el medio de contraste con márgenes claros de tejido sano (39).

\section{Laboratorio}

Los datos del laboratorio de estudios previos señalan que los valores de la PTH pueden ser normales, aumentados o disminuidos así como el producto calciofósforo. En la piel produce lesiones necrotizantes que pueden complicarse con gangrena, infección y sepsis, lo que condiciona su pronóstico (39).

Hay comunicaciones de pacientes con calcifilaxis e insuficiencia renal crónica que se presentaron con signos y síntomas de dermatomiositis (40). Las características clínicas en la piel consisten en lesiones dolorosas constituídas por placas violáceas y moteadas (livedo reticulares) que, según su distribución, pueden ser (41) de tipo distal -en miembros inferiores (90\% de los casos) como piernas, tobillos y dedos, que, en general, son de buen pronóstico y alcanzan restitución ad integrum-, de tipo proximal -afectan abdomen, genitales, muslos y hombros, suelen ir seguidas de úlceras penetrantes gangrenosas y son de mal pronóstico por complicarse con sepsis-.

También se han comunicado casos de calcifilaxis asociados con sangrado gastroduodenal masivo debido a defectos en la arteria mesentérica superior y sus tributarias (42). Ello en parte puede obedecer a su particular afinidad por el tejido elástico, siendo las localizaciones viscerales más comunes: pulmón, estómago, riñón, piel, corazón y sistema vascular (43). De esto se desprenden otros síntomas y signos menos frecuentes como: arritmias secundarias a calcificaciones miocárdicas, enfermedades restrictivas pulmonares y pancitopenias secundarias a fibrosis medular.

Al realizar las pruebas de histología, se observa un depósito de calcio en la capa media de arterias de pequeño y mediano calibre de la dermis y tejido subcutáneo, que puede estar acompañado de hiperplasia de la íntima, fibrosis, trombosis, calcificación extravascular e infiltrado inflamatorio en tejido subcutáneo, predominantemente lobulillar. Sumado a esto, se pueden ver imágenes de dermatitis de estasis y/o paniculitis esclerosante. De los anteriores, la calcificación de la capa media de los vasos es el primer hecho detectable y es un hallazgo universal en todas las fases, incluso en ausencia de otras alteraciones patológicas (17). En ocasiones sólo se calcifican, o lo hacen preferentemente los vasos hipodérmicos, por lo que la biopsia debe realizarse siempre en profundidad, llegando hasta tejido celular subcutáneo. A veces es necesario realizar cortes seriados de la pieza para poder encontrar los hallazgos histológicos característicos (12).

\section{Pronóstico}

En cuanto al pronóstico de la calcifilaxis, si bien es una patología poco frecuente, cuando se presenta produce un cuadro clínico grave que a pesar de los avances en las técnicas dialíticas tiene pésimo pronóstico, con morbilidad asociada a infecciones y a mortalidad en pocos meses por compromiso séptico y disfunción de los órganos afectados. En la mayoría de los casos, entre el $60 \%$ y el $80 \%$, llevará a la muerte del paciente antes de 10 meses de diagnosticado el 
ISSN-PRINT

1794-9831

E-ISSN 2322-7028

Vol. 15 No. 1

Ene - Jun 2018

Cúcuta, Colombia cuadro (44) y casi siempre la causa final será séptica, sobre todo secundaria a infecciones cutáneas. Algunos autores hacen una distinción a efectos pronósticos, diferenciando entre dos grupos según la localización de las lesiones: si se ubican sólo en zonas distales (piernas, antebrazos, manos y pies) puede producirse gangrena y ser necesaria la amputación quirúrgica, aunque la supervivencia del paciente es mayor. Si predominan en zonas proximales (tronco, muslos, brazos), la tasa de mortalidad es mucho más alta $(5,18)$.

\section{Cuidados de enfermería}

Dentro de la búsqueda realizada, no se encontraron cuidados específicos relacionados con la disciplina enfermera para el manejo de estos pacientes. Apenas, en algunos artículos, se abordaron cuidados generales para el paciente y su patología, pero no de forma directa las lesiones a las que está expuesto, las cuales presentan diversos problemas, entre ellos el diagnóstico diferencial y, a su vez, el tratamiento, lo cual genera que sea mucho más compleja la atención de las mismas, se aumenten las complicaciones derivadas de un manejo inadecuado, se dé una disminución en la calidad de vida del paciente y, por ende, se produzca más rápido la muerte.

\section{El proceso de enfermería como estrategia para atender pacientes con lesiones de tipo calcifilaxia}

En la literatura científica analizada no se encuentran de forma directa cuidados realizados por el profesional de enfermería a este paciente. De hecho, la mayoría de publicaciones solamente abordan cuidados generales, aunque se reconoce que los cuidados de enfermería son de vital importancia en los pacientes que presentan una úlcera de cualquier tipo, para quienes apenas se contemplan aspectos generales y no de forma directa con las lesiones, lo cual complica el cuidado que brinda el profesional, pues no existe un consenso claro al respecto, aumentando la dificultad del manejo. En el caso de los pacientes renales, el abordaje debe hacerse teniendo en cuenta las características específicas de este tipo de enfermos.

Las úlceras por calcifilaxis suponen un reto para el profesional enfermero, dado que tienen una baja incidencia, conllevan un mal pronóstico y, por ende, traen un desconocimiento general de sus principales características. Además, el profesional debe tener en cuenta que estas lesiones son muy dolorosas para el paciente, tienen un riesgo alto de infección y responden de forma irregular a las curas tradicionales, lo que requiere que el diagnóstico y el tratamiento se deban realizar de forma rápida, ya que, en muchas ocasiones, estas úlceras son consideradas como lesiones de origen vascular y se les proporciona un tratamiento totalmente diferente que genera más complicaciones en el paciente, empeorando el pronóstico. Por ello, se proponen algunos aspectos importantes que debe contemplar el profesional encargado del cuidado de estos pacientes.

En cuanto a las úlceras, en la exploración macroscópica, éstas se muestran como una placa necrótica con bordes irregulares, tejido esfacelar, edema perilesional y abundante exudado. Es importante recordar que evolucionan rápidamente de lívedo reticularis a placas necróticas escarificadas. El tratamiento debe desarrollarse con un enfoque multidisciplinar, ya que no existe un tratamiento totalmente eficaz.

Las úlceras con tejido necrótico tienen alto riesgo de sobreinfección y, en el ámbito intrahospitalario, el porcentaje de colonización por pseudomona es del $50 \%$, razón por cual debe tenerse muy en cuenta este aspecto cuando el paciente se encuentra en este medio (18). Se ha referido que el patrón de distribución podría predecir el pronóstico, siendo éste mejor para las lesiones distales o acral (dedos de manos, pies y pene), que las que involucran segmentos proximales como muslos, glúteos, abdomen y tronco.

En la actualidad, el tratamiento de la calcifilaxis se basa en:

1) La eliminación de factores implicados en su aparición y desarrollo.

2) El tratamiento del componente inflamatorio de las lesiones y los depósitos de calcio.

3) Las curas intensivas de las úlceras, utilizando el sistema Vacuum Assisted Closure (VAC) (4) y la cámara hiperbárica (7)

4) El tratamiento de los episodios infecciosos de las lesiones.

5) El tratamiento del dolor y el mantenimiento de un buen estado nutricional.

De las anteriores medidas terapéuticas, las que mejor resultado han demostrado y que han sido eficaces, aunque de forma irregular, incluyen: 
- Normalización de la calcemia, fosfatemia $y$ producto fosfocálcico: fundamentalmente con el empleo de captores no cálcicos, bajas concentraciones de calcio en el baño de diálisis y evitar el uso de metabolitos activos de vitamina D.

- Control del hiperparatiroidismo secundario: con calcimiméticos o paratiroidectomía, ante la falta de rápida respuesta a los calcimiméticos.

- Cámara hiperbárica: con objeto de aumentar la PO2 tisular y, con ello, la producción de fibroblastos y de colágeno que pueden favorecer la angiogénesis.

- Bifosfonatos: que poseen un potente efecto inhibitorio sobre la actividad osteoplástica y resorción ósea. La inhibición de la resorción ósea disminuye la concentración de calcio en la sangre y, con ello, reduciría la tendencia de los núcleos minerales para formarse y crecer en las paredes arteriales. Por otro lado, los bifosfonatos podrían inhibir la secreción de citoquinas proinflamatorias a nivel de la pared vascular, lo cual podría contribuir a mejorar el cuadro.

- Aplicación de tiosulfato sódico endovenoso: se ha mostrado eficaz aunque se desconoce el mecanismo de acción, que probablemente se relaciona con la disolución de las sales de calcio depositadas en los vasos. Se administra como solución al $25 \%$, $25 \mathrm{~g} / 1,73 \mathrm{~m} 2$ a pasar durante la hora final de cada sesión de hemodiálisis hasta la resolución del cuadro

- Curación de las úlceras: también se han utilizado larvas estériles en la granulación y epitelización de las úlceras, con buenos resultados en algunos pacientes

La prevención y el diagnóstico precoz de la calcifilaxis son fundamentales para disminuir su incidencia (910). Se debe identificar a los pacientes de alto riesgo, dentro de los que se cuentan: mujeres, pacientes obesos, diabéticos, malnutridos, tratamiento con anticoagulantes orales, inmunosupresores y realizar un control estricto de los valores séricos de Ca y de $\mathrm{P}$, destacando el papel de los captores de $\mathrm{P} \sin \mathrm{Ca}$, como el carbonato de lantano y el sevelamer, el paricalcitol y los calcimiméticos.

Estudios sobre calidad de vida relacionados con la salud han mostrado, de forma sistemática, que el dolor disminuye de manera importante si se sigue un tratamiento eficaz que induzca a la curación. El tratamiento eficaz del dolor es esencial en la calidad de la atención sanitaria y suele depender de la capacidad de los profesionales sanitarios para comprender el impacto que dichos factores tienen sobre el paciente (45).

El principal cuidado que se debe brindar al paciente con calcifilaxia es evitarle el dolor, que es de tipo neuropático -factor importante en el desarrollo del dolor crónico-, por lo que es importante que el profesional lo identifique y derive a un especialista que pueda tratarlo o que se ingrese al paciente a un programa de dolor y cuidados paliativos. Además, el enfermo experimenta aumento del dolor habitualmente durante las curaciones, en la retirada y/o aplicación de apósitos, la limpieza de la herida y el desbridamiento (46). Es importante manipular estas heridas con suavidad, pues cualquier contacto puede producir dolor y, a la hora de elegir un apósito, es necesario tener en cuenta: que sea el apropiado para la herida, que mantenga un medio ambiente húmedo para reducir las fricciones en la superficie, que minimice el dolor y los traumatismos durante su retirada y que pueda permanecer en su sitio durante largos periodos de tiempo (47).

En cuanto al tratamiento farmacológico, es importante la instauración de una analgesia adecuada y suficiente para cada paciente y la revisión de la pauta de analgésicos. Los autores recomiendan que el analgésico tarde poco en alcanzar el efecto máximo, que se ajuste fácilmente a los requisitos del cambio y produzca los mínimos efectos secundarios posibles. Además, la aplicación tópica de analgésicos locales puede ayudar a aliviar la intensidad del dolor.

Una de las principales complicaciones en el paciente con lesiones tipo calcifilaxia es la infección, asunto que se convierte en un tema de actualidad por diferentes motivos: el aumento de la prevalencia, la gravedad de las consecuencias clínicas y epidemiológicas, la amenaza de los microorganismos resistentes, la importancia del uso racional de los antibióticos y la necesidad de mejorar el diagnóstico y la elección de las nuevas opciones terapéuticas. Por sí solo, el estudio microbiológico no es un método fidedigno para diagnosticar la infección de las heridas, que requiere una consideración completa del paciente. La adquisición de especies bacterianas por una herida puede producir tres resultados definidos: contaminación, colonización e infección. Una situación adicional es la descrita 
ISSN-PRINT

1794-9831

E-ISSN 2322-7028

Vol. 15 No. 1

Ene - Jun 2018

Cúcuta, Colombia como "colonización crítica", consistente en un estadio intermedio entre la colonización benigna y la infección manifiesta en la herida. Se manifiesta como el retraso en la cicatrización "herida indolente" debido al desequilibrio bacteriano y aparecen algunos signos clínicos como color rojo brillante en el lecho de la úlcera y aumento del exudado (48).

La identificación precoz de la infección de una herida permite aplicar la intervención antimicrobiana adecuada, puesto que la infección interrumpe siempre el proceso de cicatrización normal y requiere de un diagnóstico y un tratamiento eficaz. Cuando es necesario reducir la carga microbiana, la elección del apósito antimicrobiano debe basarse en su capacidad para controlar el aumento del exudado, eliminar el tejido necrótico, reducir el mal olor, adaptarse al tamaño y forma de la herida, realizar las funciones de preparación de la herida, satisfacer las expectativas del paciente y cumplir los objetivos del tratamiento.

El uso de apósitos que contienen plata en el tratamiento de las heridas crónicas es cada vez más frecuente. Existen pruebas de que la plata puede tener propiedades antiinflamatorias. Además, el uso de apósitos de tipo Hydrofiber ${ }^{\circledR}$, que contienen plata, potencia la eliminación y la inactivación de microorganismos al retenerlos en la matriz del apósito. Ahora bien, existen indicios de resistencia bacteriana a la plata, razón por la cual la exposición a la misma podría favorecer la aparición de microrganismos resistentes y esto podría explicar, en gran parte, el predominio de bacterias intrínsecamente resistentes (49).

El exudado en las heridas se produce en respuesta a una interacción entre la etiología de la herida, la fisiología de la cicatrización de la herida, el ambiente de la herida y los procesos patológicos agravantes de la misma. En la actualidad, se sabe que el exudado ayuda en la cicatrización al evitar que se seque el lecho ulceral, en la migración de las células reparadoras de los tejidos al aportar nutrientes esenciales para el metabolismo celular, a permitir la difusión de factores inmunitarios y de crecimiento, ayudando a reparar el tejido desvitalizado o lesionado. Aun así, se puede convertir en un problema para el paciente y el personal enfermero, cuando la cantidad producida y/o su composición retrasan o impiden la cicatrización. Por todo esto, el exudado debe gestionarse en aras de maximizar sus efectos beneficiosos para la herida y para el paciente, mediante una correcta valoración y aplicación de cuidados enfermeros, reduciendo al mínimo los efectos perjudiciales y maximizando los efectos positivos. La importancia del exudado en la cicatrización de las heridas hace que el objetivo habitual consista en lograr un lecho húmedo pero no macerado. El manejo eficaz del mismo precisa de la elaboración de planes de cuidados individualizados, adecuados a cada paciente y que aborde los problemas relacionados con este, tales como el mal olor y el dolor. Los apósitos representan la principal opción para manejar el exudado, por lo que el personal enfermero debe decidir sobre la necesidad de modificar o mantener su régimen de aplicación. El apósito de Hydrofiber ${ }^{\circledR}$ es un apósito adaptable y muy absorbente que se transforma en un gel suave al contacto con el exudado, lo que mantiene un medio húmedo adecuado para favorecer el proceso de cicatrización y el desbridamiento autolítico, sin dañar el tejido neoformado.

\section{Conclusiones}

- Las calcificaciones vasculares y de tejidos blandos es un hallazgo bastante frecuente en los pacientes urémicos, pero estos depósitos cálcicos ocasionalmente producen insuficiencia vascular y necrosis secundaria a cambios isquémicos. Los mecanismos de calcificación conocidos son el hiperparatiroidismo, la hiperfosforemia, la hipervitaminosis D y el tratamiento esteroideo, entre otros. Sin embargo, se desconocen los factores que, en un momento determinado, desarrollan calcificaciones vasculares de tal magnitud que producen lesiones isquémicas periféricas.

- La calcifilaxis es un cuadro infrecuente, generalmente asociado con el hiperparatiroidismo secundario en los pacientes urémicos con múltiples factores de riesgo asociados. Por otra parte, no existe, en la actualidad, un tratamiento totalmente eficaz para esta complicación (50).

- Lo anterior implica que el tratamiento debe ser multidisciplinar e incluir el control del hiperparatiroidismo, la normalización del producto $\mathrm{Ca} / \mathrm{P}$, el manejo efectivo del dolor y un cuidado integral de la úlcera, evitando la sobreinfección, la necrosis o la extensión que provoca la necesidad 
de un tratamiento quirúrgico agresivo. También se demuestra que es de vital importancia la planificación de cuidados de enfermería integrales, encaminados a prevenir complicaciones y mejorar la calidad de vida del paciente. Las úlceras por calcifilaxis originan molestia en el paciente debido al dolor neuropático que producen y por la aplicación de las curas con los cambios de apósitos.

- Según la evidencia encontrada, los pacientes con calcifilaxia son, en la actualidad, un reto para los profesionales de la salud ya que no existen cuidados específicos para las diferentes alteraciones que presentan. A esto se suma el desconocimiento general de la patología, situación que provoca que la atención brindada tenga complicaciones y, en ocasiones, resulte inadecuada, lo que hace que estos pacientes sean un reto para los profesionales enfermeros.
- Después de utilizar y aplicar la metodología GRADE se puede concluir que los estudios analizados arrojan una calidad de evidencia alta, sobre todo en los tratamientos propuestos con calcio y fósforo, además de otros tratamientos complementarios que se usan en estos pacientes. En cuanto al cuidado de las lesiones, se observa que es demasiado básico pero muestra alguna efectividad sobre todo cuando el paciente es identificado a tiempo. Por ello, es necesario que los profesionales de enfermería diseñen planes de cuidado específicos para este tipo de lesiones y así puedan contar con herramientas más efectivas para la atención de alguna de estas lesiones.

\section{Conflicto de Intereses}

Los autores declaran no tener ningún conflicto de intereses.

\section{Referencias bibliográficas}

1. Angelis MW, Wong LL, Myers SA, Wong LM. Calciphylaxis in patients on hemodialysis: a prevalence study. Surgery 1997; 122:1083-1089.

2. Verdalles Guzmán U, Verde E, García de Vinuesa S, Goicoechea M, Mosse A, et al. Calcifilaxis: complicación grave del síndrome cardio-metabólico en pacientes con enfermedad renal crónica terminal (ERCT). Nefrología (Madrid) [Internet] 2008. [consultado 2016 Sept. 16]; 28:32-6 Disponible en http:/www.revistanefrologia.com/es-publicacionnefrologia-articulo-calcifilaxis-complicacion-gravedel-sindrome-cardio-metabolico-pacientes-conenfermedad-renal-X0211699508033201

3. Munar MA, Bernabéu R, Morey A, Alarcón A, Gascó J, et al. A propósito de un caso de lesiones cutáneas y calcifilaxis. Nefrología (Madrid.) [Internet] 2001. [consultado 2016 Sept. 16]; 21:501-4. Disponible en http://www.revistanefrologia.com/es-publicacion-nefrologia-articulo-aproposito-uncaso-lesiones-cutaneas-calcifilaxis-X0211699501013882

4. Gómez de la Fuente E, Álvarez JG, Naza E, Palencia S, et al. Calcifilaxis en pacientes dializados. [Internet] 2004. [consultado 28 agosto 2016]; 95(3):66-70. Disponible en: http://www.actasdermo.org/ es/linkresolver/calcifilaxis-pacientes-dializados/13060644/

5. Mazhar AR, Johnson RJ, Giller D, Stivelman JC, Ryan MJ, et al. Risk factors and mortality associated with calciphylaxis in end-stage renal disease. Kidney Int 2001; 60: 324-332.

6. Esteve, V. Almirall, J. Luelmo, J. Sáez, A. Andreu, X. García, M. Arteriolopatía urémica calcificante (calcifilaxis): incidencia, formas de presentación y evolución. Nefrología. [Internet] 2007. [consultado 2016 Sept. 16]; 27(5):599-604. Disponible en: http://www.revistanefrologia.com/es-publicacionnefrologia-articulo-arteriolopata-urmica-calcificante-calcifilaxis-incidencia-formas-de-presentacin-yevolucin--X021169950702170X

7. Prados Soler MC, Pino MD, Garófano López R, Moriana Domínguez C. Calcifilaxis severa en paciente en hemodiálisis. Dial Traspl. [Internet] 2010; [consultado 2016 Sept. 16]; 31:76-8. Disponible en: http://www.elsevier.es/es-revista-dialisis-trasplante-275-articulo-calcifilaxis-severa-pacientehemodial isis-S1886284510000524

8. Polaina Rusillo M, Sánchez Martos MD, Biechy Baldan MM, Liebana Cañada A. Calcifilaxis. Semin 
ISSN-PRINT

$1794-9831$

E-ISSN 2322-7028

Vol. 15 No. 1

Ene - Jun 2018

Cúcuta, Colombia
Fund Esp Reumatol [Intenet] 2009. [consultado 2016 Sept. 16]; 10:124-7. Disponible en: http://www. elsevier.es/es-revista-seminarios-fundacion-espanola-reumatologia-274-articulocalcifilaxis-

9. Rodríguez Villarreal I, Callejas R, Sánchez M, Laso N, Gallar P, et al. Calcifilaxis o arteriolopatía urémica calcificante: combinación de tratamientos. NefroPlus [Internet] 2010. [consultado 2016 Sept. 15]; 3:39-44. Disponible en : http://www.revistanefrologia.com/es-publicacion-nefroplus-articulocalcifilaxis-o-arteriolopatia-uremicacalcificante-combinacion-tratamientos-X1888970010000760

10. Pérez JE, Vargas JG, Echeverri JE, Rodríguez PV, Pulido JA. Calcifilaxis y enfermedad renal crónica. [Internet] 2011. [Consultado 2016 agosto 28]; 36(3):149-152. Disponible en: http://www.scielo.org. co/pdf/amc/v36n3/v36n3a08.pdf

11. Piña Simón D, González Horna A, Gálvez Serrano MC, Marco García B, Rubio González E. Actuación de enfermería ante un paciente con calcifilaxis. Rev Soc Esp Enferm Nefrol [Internet] 2010. [Consultado 2016 agosto 28]; 13(3):205-208 Disponible en: http://scielo.isciii.es/pdf/nefro/v13n3/caso_clinico1. pdf

12. Picazo M, Bover J, De La Fuente J, Sans R, Cuxart M, Matas M. Larvas estériles como coadyuvantes al tratamiento local en una paciente con calcifilaxis proximal. Nefrología. [Internet] 2005. [Consultado 2016 agosto 28]; 25:559-62.Disponible en: http://www.revistanefrologia.com/es-publicacionnefrologia-articulo-larvas-esteriles-como-coadyuvantes-al-tratamiento-localuna-pacientecon-X0211699505018788

13. Campoy MV, Ibáñez MJ, Luna A, Matamoros E, Michelena MA, et al. A propósito de tres casos. Arch. Argent. Dermatol. [Internet] 2014. [Consultado 2016 agosto 28] Disponible en: http://www. archivosdermato.org.ar/Uploads/Enprensa\%20Campoy-Calcifilaxis.pdf

14. Edwards RF, Jaffe W, Arrowsmith J, Henderson HP. Calciphylaxis: a rare limb and life-threatening cause of ischaemic skin necrosis and ulceration. Br J Plastic Surg. 2000; 53:253-255

15. González Alvarado A. Arteriolopatía urémica calcificante (Calcifilaxis) genital: análisis del diagnóstico, tratamiento y supervivencia clínica. Serie de casos. [Tesis de grado Especialista en Urología]. XalapaEnríquez: Universidad Veracruzana; 2014.

16. Rojas Estrada JJ, Castelo Villalón X, Sat FA, Semanat Vaillant D. Arteriolopatía calcificante urémica. Nefroplus. [Internet] 2010. [Consultado 2016 agosto 28] 3(3):39-44. Disponible en: http://www.bvs. sld.cu/revistas/med/vol50_2_11/med11211.htm

17. Polaina M, Pérez MP, Carrillo AM, Ramírez C, Borrego J, Liébana A. Necrosis cortical renal secundaria a microangiopatía trombótica en el contexto de crisis blástica de leucemia aguda promielocítica. Nefrología. [Internet]. 2013 [citado 2017 Jun. 05]; 33(6): 845-848. Disponible en: http://dx.doi. org/10.3265/Nefrologia.pre2013.Sep.12162

18. Manterola CD, Zavando DM. Cómo interpretar los "Niveles de Evidencia" en los diferentes escenarios clínicos. Rev. Chil Cir [revista en Internet]. 2009 dic. [Consultado 30 octubre 2016]; 61(6):582-595. Disponible en: http://www.scielo.cl/scielo.php?script=sci_pdf\&pid=S0718-40262009000600017\&ln $\mathrm{g}=$ es\&nrm=iso\&tlng=es

19. Curbelo Rodríguez L, Velazco Oiz Y, Triana del Toro T, Hernández Rodríguez M. Calcifilaxis. Reporte de dos casos. Archivo Médico de Camagüey. Internet] 2006 [Consultado 2016 agosto 28]; 10(5). Disponible en: http://www.redalyc.org/pdf/2111/211117629015.pdf

20. Rodríguez L, Di Martina Ortiz B, Contreras R, Rodríguez M, Knopfelmacher O, Bolla L. Calcinosis cutis metastásica: Calcifilaxis (arteriolopatía urémica calcificada). A propósito de un caso. Our Dermatology Online, [Internet] 2013. [Consultado 16 Mar. 2017]; 4(3):358-360. Disponible en: http:// www.odermatol.com/odermatology/32013/26.Calcinosis-RodriguezL.pdf

21. Fader DJ, Kang S. Calciphylaxis without renal failure. Arch Dermatol. 1996; 132(7):837-8.

22. Anderson DC, Stewart WK, Piercy DM. Calciphylaxis panniculitis with fat and skin necrosis in a case of uremia with autonomous hyperparathyroidism. Lancet. 1968; 2:323-325.

23. Grimaudo V, Gueissaz F, Hauert T. Necrosis of skin induced by coumarin in a patient deficient in protein S. Br Med J 1989; 298: 233-234.Ahmed, S.; O’Neill, K.; Hood, A.: Calciphylaxis is associated with hyperphosphatemia and increased osteopontin expression by vascular smooth muscle cells. Am J 
24. Ahmed S, O'Neill K, Hood A. Calciphylaxis is associated with hyperphosphatemia and increased osteopontin expression by vascular smooth muscle cells. Am J Kidney Dis 2001; 37: 1267-1276

25. Brítez M, Schupp E, Romero M, Arévalos N, Daponte F, et al. Reporte de caso. Calcifilaxis en un paciente en Hemodiálisis. Estudio clínico anatomopatológico. [Internet] [Consultado 2016 agosto 28] Disponible en: http://www.ins.gov.py/revistas/index.php/anales/article/view/241/195

26. Verdalles Guzmán U, De la Cueva P, Verde E, García de Vinuesa S, Goicoechea M, Mosse A, et al. Calcifilaxis: complicación grave del síndrome cardio-metabólico en pacientes con enfermedad renal crónica terminal (ERCT). Nefrología. 2008; 28(1):32-6.

27. Guerra G, Shah RC, Ross EA. Rapid resolution of calciphylaxis with intravenous sodium thiosulfate and continuous venovenous haemofiltration using low calcium replacement fluid: case report. Nephrology Dial Transplant. 2005; 20(6):1260-2.

28. Pérez Marín Jc, Peña Quintana $P$, Suárez Cabrera M, Calvo Hernández LM, Acosta E, et al. Ulceras por Calcifilaxia sin Insuficiencia Renal. Canar Méd Quir. 2003;1(2):4-6

29. Nigwekar SU, Wolf M, Sterns RH, Hix JK. Calciphylaxis from nonuremic causes: a systematic review. Clin J Am Soc Nephrol. 2008; 3(4):1139-43.

30. García S, Gómez ML, Rojas Bilbao EA.Calcifilaxis en un paciente sometido a hemodiálisis. Factores de riesgo asociados y revisión bibliográfica. Arch Argent Dermatol. 2002; 52: 239-244

31. Pérez JE, Vargas JG, Echeverri JE, Rodríguez PV, Pulido JA. Calcifilaxis y enfermedad renal crónica. Acta Med Colomb 2011; 36: 149-152.

32. Rodríguez L, Di Martina Ortiz B, Contreras R, Rodríguez Masi M, Knopfelmacher O, Bolla de Lezcano L. Calcinosis cutis metastásica: calcifilaxis (arteriolopatía urémica calcificada). A propósito de un caso. Our Dermatol Online 2013; 4: 358-360.

33. Marrón B, Coronel F, López Bran E, Barrientos A. Calcifilaxia: una patogenia incierta y un tratamiento controvertido. Nefrología 2001; 21: 596-600.

34. Cohen GF, Vyas NS. Sodium thiosulfate in the treatment of calciphylaxis. J Clin Aesthet Dermatol 2013; 6: 41-44.

35. Prados MC, Pino MD, Garófano López R, Moriana C. Calcifilaxis severa en paciente con hemodiálisis. Dial Traspl 2012; 31: 76-78.

36. Markova A, Lester J, Wang J, Robinson Bostom L. Diagnosis of common dermopathies in dialysis patients: a review and update. Semin Dial 2012; 25: 408-418

37. Nikko A, Dunningan M, Cockerelle CJ. Calciphylaxis with histologic changas of pseudoxanthoma elasticum. Am J Dermatopathol. 1996; 18: 396-399

38. Kant S, Glueck H. Protein S deficiency and skin necrosis associated with continuous ambulatory peritoneal dialysis. Am J Kidney Dis. 1992; 19: 264-271.

39. Chan YL, Mahony JF, Turner JJ, Posen S. The vascular lesions associated with skin necrosis in renal disease. Br J Dermatol. 1983; 109: 85-95.

40. Flanigan $\mathrm{K}$, Bromberg M. Calciphylaxis mimicking dermatomyositis: ischemic myopathy complicating renal failure. Neurology. 1998; 51: 1634-1640.

41. Oh DH, Eulau D, Tokugawa DA, McGuire JS, Kohler S. Five cases of calciphylaxis and review of the literatura. J Am Acad Dermatol 1999; 40: 979-987

42. Brown D, Denney C, Burns D. Systemic calciphylaxis associated with massive gastrointestinal hemorrhage. Arch Pathol Lab Med 1998; 122: 656-659.

43. Barbur M, Kurjak M. Systematic calciphylaxis in chronic renal failure: fulminant course after kidney transplantation. Pathologe 1997; 18: 453-458

44. Rodríguez Villarreal I, Callejas R, Sánchez M, Laso N, Gallar P, Ortega O, et al. Calcifilaxis o arteriolopatía urémica calcificante: combinación de tratamientos. NefroPlus [Internet] 2010; [consultado 2016 Sept. 15]; 3:39-44. Disponible en: http://www.revistanefrologia.com/es-publicacion-nefroplus-articulocalcifilaxis-o-arteriolopatia-uremicacalcificante-combinacion-tratamientos-X1888970010000760

45. Briggs M, Chronic wounds, non-healing wounds or a possible alternative? The ewma Journal 2010; 
ISSN-PRINT

1794-9831

E-ISSN 2322-7028

Vol. 15 No. 1

Ene - Jun 2018

Cúcuta, Colombia
10(3):21-23.

46. Bulechek GM, Butcher HK, McCloskey JD (eds.). Clasificación de Intervenciones de Enfermería (nic). $5^{\mathrm{a}}$ ed. Barcelona: Elsevier; 2009

47. Manterola CD, Zavando DM. Cómo interpretar los "Niveles de Evidencia" en los diferentes escenarios clínicos. Rev. Chil Cir [revista en Internet]. 2009 dic. [acceso: 30 oct. 2014]; 61(6):582-595. Disponible en: http://www.scielo.cl/scielo.php?script=sci pdf\&pid=S0718-40262009000600017\&lng=es\&nrm= iso\&tlng=es

48. Manterola CD, Zavando DM. Cómo interpretar los "Niveles de Evidencia" en los diferentes escenarios clínicos. Rev. Chil Cir [revista en Internet] 2009. [Consultado 30 oct. 2016]; 61(6):582-595. Disponible en: http://www.scielo.cl/scielo.php?script=sci_pdf\&pid=S0718-40262009000600017\&lng=es\&nrm= iso\&tlng $=\mathrm{es}$

49. Thoroddsen A, Ehnfors M. Nursing specialty knowledge as expressed by standardized nursing languages. Int J Nurs Terminol Classif. 2010; 21(2):69-79. 